\title{
Morphological Characterization of Twenty One Sweet Pepper (Capsicum annuum L.) Genotypes Collected from Native and Alien Sources
}

\author{
Jannatul Ferdousi, Mohammad Zakaria, Md. Azizul Hoque, Satya Ranjan Saha, \\ Nasrin Akter Ivy, and Md. Iqbal Hossain
}

\section{ABSTRACT}

Sweet pepper is one of the most important nutritious vegetable and its demand is increasing day by day in Bangladesh indicating need to characterize and assess morphological variability for varietal improvement programme. Twenty-one sweet pepper genotypes from native and alien sources were characterized for twenty-six morphological traits using vegetative and reproductive appearances at Bangabandhu Sheikh Mujibur Rahman Agricultural University, Gazipur, Bangladesh from October 2018 to March 2019. Marked variation was exhibited among twenty-six qualitative traits (26) studied. Twenty-two (22) characters showed undeniable variation among the genotypes. The presence of higher percentage $(61.90 \%)$ of light purple color at node were observed indicated high amount of anthocyanin content. Leaf shape is used as genotypes identifier at vegetative stage and herein three types of leaves were found with dark green color $\mathbf{( 7 6 . 1 9 \%}$ ) that is highly correlates with yield. In case of flower, same level of stigma exertion $(\mathbf{6 1 . 9 0 \%})$ with $100 \%$ white color corolla indicates higher number of fruit sett were exhibited. Entire genotypes exerted one or more exclusive characters especially fruit shape and color in Bangladesh perspective which could be used as an important breeding tools. Fruit color was observed in various categories at intermediate and mature stage as for instance yellow, green, purple, orange, red etc. in addition higher percentage of blocky fruit shape $\mathbf{( 3 8 . 0 9 \%}$ ) were observed and these are the consumer fascinating attributes of sweet pepper. However, selection of genotypes with desirable morphological trait can be used for their exploitation of future breeding programme.

Keywords: Fruit shape and color, Morphological variation, Qualitative trait, Sweet pepper, Varietal improvement.

\section{INTRODUCTION}

Sweet pepper or Bell pepper is a cultivar group of the species Capsicum annuum L. an important vegetable crop, grown worldwide for its delicate taste, pleasant flavour and color. Capsicum is a genus of flowering plants in the nightshade family Solanaceae. Though the genus Capsicum contains about 20 species, till now only five domesticated species viz. Capsicum annum, C. frutescens, C. chinense, $C$. baccatum and $C$. pubescens are recognized [1]. All cultivated species of sweet pepper have $2 n=24$ chromosomes [2]. As a food, sweet pepper has high nutritive value as it contains 175
Published Online: September 04, 2021

ISSN: 2684-5199

DOI: $10.24018 /$ ejbio.2021.2.5.254

Jannatul Ferdousi *

Assistant Professor, Dept. of Horticulture, Sylhet Agricultural University, Sylhet, Bangladesh.

(e-mail: ferdousi.hort@sau.ac.bd)

Mohammad Zakaria

Professor, Dept. Horticulture, Bangabandhu Sheikh Mujibur Rahman Agricultural University, Gazipur-1706, Bangladesh.

(e-mail: zakaria1963@yahoo.com)

Md. Azizul Hoque

Professor, Dept. Horticulture, Bangabandhu Sheikh Mujibur Rahman Agricultural University, Gazipur-1706, Bangladesh.

(e-mail: azizul@bsmrau.edu.bd)

Satya Ranjan Saha

Professor, Dept. of Agroforestry and Environment, Bangabandhu Sheikh Mujibur Rahman Agricultural University, Gazipur-1706, Bangladesh.

(e-mail: satya@bsmrau.edu.bd)

Nasrin Akter Ivy

Professor, Dept. of Genetics \& Plant Breeding, Bangabandhu Sheikh Mujibur Rahman Agricultural University, Gazipur1706, Bangladesh.

(e-mail: ivy.bsmrau@yahoo.com)

Md. Iqbal Hossain

PhD Fellow, Dept. of Agroforestry and Environment, Bangabandhu Sheikh Mujibur Rahman Agricultural University, Gazipur-1706, Bangladesh.

(e-mail: iqbalsaubd@gmail.com)

*Corresponding Author mg ascorbic acid, 870 I.U. vitamin A, $1.29 \mathrm{mg}$ protein, 0.06 $\mathrm{mg}$ thiamine, $0.03 \mathrm{mg}$ riboflavin, $0.55 \mathrm{mg}$ niacin and $11 \mathrm{mg}$ calcium per $100 \mathrm{~g}$ edible fruit [3]. Peppers are also well known for its high content in bioactive compounds and strong antioxidant properties including neutral phenolic compounds or flavonoids called quercetin, luteolin, and capsaicinoids [4]. The nutritional status of Bangladeshi people is a matter of great concern as more than half of the populations have been suffering from malnutrition [5], [6]. So, consumption of sweet pepper can relieve the suffering from malnutrition of Bangladeshi people to some extent, because they provide more vitamin, minerals, protein, and strong antioxidant 
properties.

Sweet pepper has been grown in Bangladesh for nearly two decades and it is a small scale cultivated vegetable in Bangladesh. According to the Ministry of Agriculture, Bangladesh [7] capsicum covers only 8 acres of land in the year 2018-2019 with a production of 11 MT. Though it has a good economic importance, but growers are not able to produce good quality capsicum with high productivity in Bangladesh [8]. In Bangladesh only two open pollinated sweet pepper varieties i.e., BARI Mistimarich 1 and BARI Mistimarich 2 has been released by Bangladesh Agricultural Research Institute [9] for cultivation. Most of the seeds used for cultivation in Bangladesh are imported [10]. Development of high yielding varieties with good quality through the advance breeding methods is essential to meet the farmers as well as market demand.

Germplasm is referred to as a set of genotypes that can be conserved or used [11]. Bangladesh is not self-sufficient in sweet pepper germplasm to fulfill its requirements and mostly depends on alien sources. Collection of diverse germplasm and their systematic evaluation assume considerable importance in any crop improvement programme [12]. Less genetic diversity means less opportunity for the growth and innovation required to boost agriculture [11]. Evaluation of the potentialities of the indigenous and exotic germplasm is essential because promise for further improvement programme depends on the genetic diversity of the crop [13].

Morphological characterization based on qualitative traits of crops is a very crucial and essential first step in any crop improvement and breeding programme [14], [15]. The immense phenotypic diversity has helped to develop a high yielding variety and morphological or phenotypic characterization is considered as the important step in the description and classification of germplasm [16], [17]. Meanwhile, studies on morphological characterization of the collected genotypes have been widely used for the assessment of genetic diversity, breeding value and yield potential of the crop [18]-[21].

Genetic cataloguing based on standard descriptors helps to easily describe the morphological features of a genotype and thus helps exchange of information about new genotypes. Therefore, the present investigation was undertaken to characterize collected native and alien sweet pepper germplasm based on their morphological traits as well as to identify promising genotypes and traits which can be used in future breeding programme.

\section{MATERIALS AND METHODS}

\section{A. Experimental Site}

The experiment was conducted at the Vegetable Research Field of the Department of Horticulture, Bangabandhu Sheikh Mujibur Rahman Agricultural University, Gazipur, Bangladesh during October 2018 to March 2019. The location of the site is $24.090 \mathrm{~N}$ latitude and $90.260 \mathrm{E}$ longitude with an elevation of $8.2 \mathrm{~m}$ from sea level under agroecological zone AEZ-28 [22]. The field experiment was installed on a high land plot on the farm. The experimental site is located in the subtropical climate zone and is characterized by three distinct seasons: the monsoon or rainy season (May to October), the winter or dry season (November to February), and the pre-monsoon or hot season (March to April). The experimental field soil was clay loam in texture that had a $\mathrm{pH}$ of around 6.2. It belongs under Madhupur tract to the Salna Series "Shallow Red Brown Terrace" soil [23], [24].

\section{B. Plant Materials}

There were twenty one (21) sweet pepper genotypes collected from native and alien sources used in this study and displayed in Table I.

\section{Experimental Design}

The experiment was laid out in a randomized complete block design (RCBD) with three replications. A total of 63 $(21 \times 3)$ unit plots were prepared. Unit plot size was $2.5 \mathrm{~m} \times$ $1.0 \mathrm{~m}$. Each plots hosted 10 (ten) plants with $50 \mathrm{~cm} \times 50 \mathrm{~cm}$ spacing. The adjacent blocks and plots were separated from one another by $1.0 \mathrm{~m}$ and $0.80 \mathrm{~m}$ spaces, respectively. The space between two blocks and two plots were made as drain having a depth of about $20 \mathrm{~cm}$ for irrigation and rapid drain out of water. The 21 genotypes were assigned randomly in each block.

\section{Seedling Raising and Transplanting}

Seeds were soaked in water for 24 hours in order to facilitate germination and subsequently were sown on plastic trays in lines. Sowing of seeds on the tray was done at a depth of one centimeter for easy emergence [25]. Sowing was done on 22 October 2018. Six to seven days were required to start germination. When the seedlings attained 3 leaf stages, they were transferred to the polybag of $12.75 \times 10.15 \mathrm{~cm}$ size, filled with potting media comprising of soil, compost and sand $(3: 1: 1)$. The seedlings were subsequently transferred under the polyethylene shade covering with fine net to prevent from scorching sunlight as well as unexpected storm or heavy rainfall and insect infestation. The seedlings were watered thoroughly every day using fine meshed sprinkler as per on the requirement. Thirty days old seedlings were transplanted on 21 November, 2018, in well prepared pit in experimental plots.

\section{E. Manure and Fertilizers Application}

Manure and fertilizers were applied in the experimental field [8] @ cowdung 10 ton, urea 250, TSP 350, MoP 250, gypsum 110 and $\mathrm{ZnO} 5 \mathrm{~kg} / \mathrm{ha}$. Half of the quantity of cow dung was applied during final land preparation. The remaining half of cow dung, the entire quantity of TSP, $\mathrm{ZnO}$, gypsum and one third each of urea and MP were applied during pit preparation. The rest of urea and MP were applied in two equal splits, 25 and 50 days after transplanting of seedlings in the field.

\section{F. Intercultural Operations and Plant Protection Measures}

During the crop cycle, appropriate intercultural operations were performed for proper plant growth and development, such as irrigation at different growth stages, weeding, soil mulching and staking as and when needed. Different types of insect infestation were occurred during the experimental period such as mites, aphids, and yellow strip armyworm. To control these vertimec, emitop and nitro @ $1 \mathrm{ml} / 1 \mathrm{~L}$ of water 
were sprayed at 7 days interval. A sex pheromone trap was set up at the fruiting stage at 12-meter intervals to prevent European Corn Borer (ECB) adult moth.

\section{G. Data Collection}

Observations of different characters were recorded from 21 sweet pepper genotype at specified stages of crop growth period when the characters under study had full expression. Five plants from each genotype were randomly selected and tagged for recording the observations. The data was taken in the form of descriptor codes assigned by IPGRI, AVRDC and CATIE (1995) [26] for the crop capsicum (Capsicum spp.). All the plant and leaf characters were observed when plants were attained at full mature stage and leaf pubescence on the younger stage. Different characters of flowers were observed immediately after anthesis. Fruit parameters were recorded by observing 10 fruits from different plants on mature fruits in the first harvest unless specified.

\begin{tabular}{cccc}
\multicolumn{4}{c}{ TABLE I: LIST OF SwEET PEPPER GENOTYPES USED IN THIS STUDY } \\
\hline Sl. No. & $\begin{array}{c}\text { Sweet pepper } \\
\text { genotypes }\end{array}$ & $\begin{array}{c}\text { Source organization of } \\
\text { collection }\end{array}$ & $\begin{array}{c}\text { Genotypes ID } \\
\text { used in this study }\end{array}$ \\
\hline 1 & AVPP 0701 & AVRDC, Taiwan & AVPP 0701 \\
2 & AVPP 0402 & AVRDC, Taiwan & AVPP 0402 \\
3 & AVPP 0504 & AVRDC, Taiwan & AVPP 0504 \\
4 & AVPP 9807 & AVRDC, Taiwan & AVPP 9807 \\
5 & AVPP 0408 & AVRDC, Taiwan & AVPP 0408 \\
6 & AVPP 1112 & AVRDC, Taiwan & AVPP 1112 \\
7 & AVPP 1115 & AVRDC, Taiwan & AVPP 1115 \\
8 & AVPP 0019 & AVRDC, Taiwan & AVPP 0019 \\
9 & BARI Mistimarich 1 & HRC, BARI & BARI M 1 \\
10 & BARI Mistimarich 2 & HRC, BARI & BARI M 2 \\
11 & Local seed & Siddique Bazar, Dhaka & LS \\
12 & White bell, P107 & England & WBP 107 \\
13 & Yellow Elf P054 & England & YEP 054 \\
14 & Purple bell P057 & England & PBP 057 \\
15 & Red bell P040 & England & RBP 040 \\
16 & PL 730/12/210/L725A & England & PL 730 \\
17 & CA 008 & HRC, BARI & CA 008 \\
18 & CA 0010 & HRC, BARI & CA 0010 \\
19 & CA 009 & HRC, BARI & CA 009 \\
20 & CA 0012 & HRC, BARI & CA 0012 \\
21 & CA 0011 & HRC, BARI & CA 0011 \\
\hline
\end{tabular}

Note: Asian Vegetable Research and Development Center (AVRDC); Horticulture Research Center (HRC); Bangladesh Agricultural Research Institute (BARI).

\section{RESUlTS AND DISCUSSION}

\section{A. Morphological Characterization}

Data from 21 sweet pepper genotypes were recorded on the basis of "Descriptors for Capsicum spp. (Capsicum annuum L.)" [26; Table II].

\section{B. Plant Growth Characteristics}

Diverse variation was found regarding eleven morphological traits of capsicum observed at appropriate stage of each genotype and are displayed in Table 3. Three types of stem color were observed such as, dominant green $(66.66 \%)$ than that of green with purple streak $(28.57 \%)$ and light green with purple streak $(4.76 \%)$. Most of the genotypes exhibited $(57.14 \%)$ cylindrical or round stem shape with strong intensity of anthocyanin coloration i.e., light purple color on nodes $(61.9 \%)$ while angled stem shape were found in case of $42.85 \%$ genotypes and $33.33 \%$ showed green color at nodes. It is [27] also observed the strong intensity of nodal anthocyanin of chilli plants. Purple color or purple color streak on chilli plants indicates the presence of high amount of anthocyanin content, which is an effective antioxidant for human body [28]. Plant growth habit was characterized as prostate, intermediate and erect, where intermediate $(61.90 \%)$ was found dominant compared to prostate (14.28\%) and erect $(23.80 \%)$. The branching habit was intermediate $(66.66 \%)$ in all the genotypes except AVPP 0504; PL 730; CA 009, 0012 (sparse) and only three genotypes (PBP 057; WBP 107; YEP 054) were dense. Through a study [29] it is observed that most of the studied genotypes of bell pepper were intermediate/compact growth habit with intermediate branching appearance.

\section{Leaf Characteristics}

Leaf shape was mainly deltoid $(57.14 \%)$; some genotypes had lanceolate $(33.33 \%)$ and ovate $(9.52 \%)$ shape leaves. These three types of leaves also found [30] in pepper accession evaluation. Through a study [14] it is investigated that dark green leaf color more frequent $(80 \%)$ while green color was less frequent $(20 \%)$ in Capsicum annuum L. genotypes. In the present investigation dark green leaf color was more $(76.19 \%)$ than green color $(23.80 \%)$. The dark green colour of leaves is generally due to presence of high chlorophyll content in the leaves which ultimately leads to increased yield. Hence, it becomes a good criterion for selection of elite cultivars group [31]. All the genotypes in the present study had entire leaf margin and sparse leaf pubescence. An experimental [29] reported the same findings in case of both leaf margin and pubescence. The leaf density was denser near about $50 \%$ genotypes $(47.61 \%)$, intermediate and sparse in case of $23.80 \%$ and $14.28 \%$ genotypes, respectively.

\section{Characterization of Reproductive Plant Part}

The most important advances obtained in the genetic improvement of plants are associated with the knowledge of their reproductive system [32] and fifteen morphological traits of reproductive organ of sweet pepper genotypes are arrayed in Table IV. Flower position and stigma exsertion highly influences the degree and mode of pollination [14]. Majority of the germplasm showed intermediate (52.38\%) flower position on the other side $38.0 \%$ and $9.52 \%$ germplasm exhibited pendant and erect type pedicel position 
during anthesis period, respectively. It is also noticed [33] almost similar results in chilli germplasm. In the present study all the genotypes were found having white corolla colour and attractive flower colour i.e., white color is a desirable trait as it helps in attracting pollinators during the pollination process [34]. Most stigmas of the flower were at the same level of stigma exertion $(61.90 \%)$ and no male sterile genotypes were found. Various types of anther color were found such as White, Yellow, Pale blue, Blue and Purple along with maximum percentage $(71.42 \%)$ of white color filament.

Fruit color of sweet pepper genotypes at different stages is one of the most desirable traits for selecting a suitable inbred line. Attractive fruit colour, lesser fruit pubescence and smooth fruit texture are the factors which determine consumer acceptability of the product. Hence, these traits become good selection criterion for a breeder [14]. In the present study it was found that fruit color at intermediate stage was green $(42.85 \%)$ and light green $(23.80 \%)$ and more prominent than yellow (19.04\%), light yellow (9.52\%) and deep purple $(4.76 \%)$. Diversified fruit color was observed at mature stage such as Lemon Yellow, Orange yellow, Orange, Red and Deep yellow. Among these, red color (66.66\%) was more dominant (Table IV). Fruit color of pepper certainly inherited by single gene [35]. For bell pepper, fruit color and fruit position are also important morphological traits to use in developing improved cultivars [36]. Wider variation was found for fruit shape. As per consumer's preference blocky fruits are more preferable and higher percentage of blocky fruits were observed $(38.09 \%)$ and others were Campanulate $(33.33 \%)$, Triangular (14.28\%), Elongate $(9.52 \%)$ and Tomato (4.72\%) (Table IV and Fig. 1). Fruits are categorized into three categories based on fruit shape at blossom end as like; Sunken (61.90\%), Pointed (23.80\%) and Blunt $(14.28 \%)$. Fruit shape at pedicel attachment was found obtuse $(14.28 \%)$, truncate $(19.04 \%)$ and lobate $(66.66 \%)$. Blocky fruit shape, lobate pedicel attachment, sunken blossom end, pendent fruit position and dark green fruit colour at maturity are desirable horticultural attributes [37]. Perfect fruit shape, size and colour along with mild taste are the main quality parameters that make the task of developing new genotypes/variety/hybrids very sticking [38]. All the germplasms were devoid of blossom end fruit appendages except PBP 057 (4.72\%). As most of the genotypes were blocky and campanulate, so corrugation at fruit cross section was dominant $(71.42 \%)$ over slightly (14.28\%) and intermediate $(14.28 \%)$ corrugation. All the genotypes had straw color seed and most of the genotypes (80.95\%) contained more than fifty $(>50)$ seeds which were measured from ten fruits in each replication and the average was considered to the number of seed per fruits.

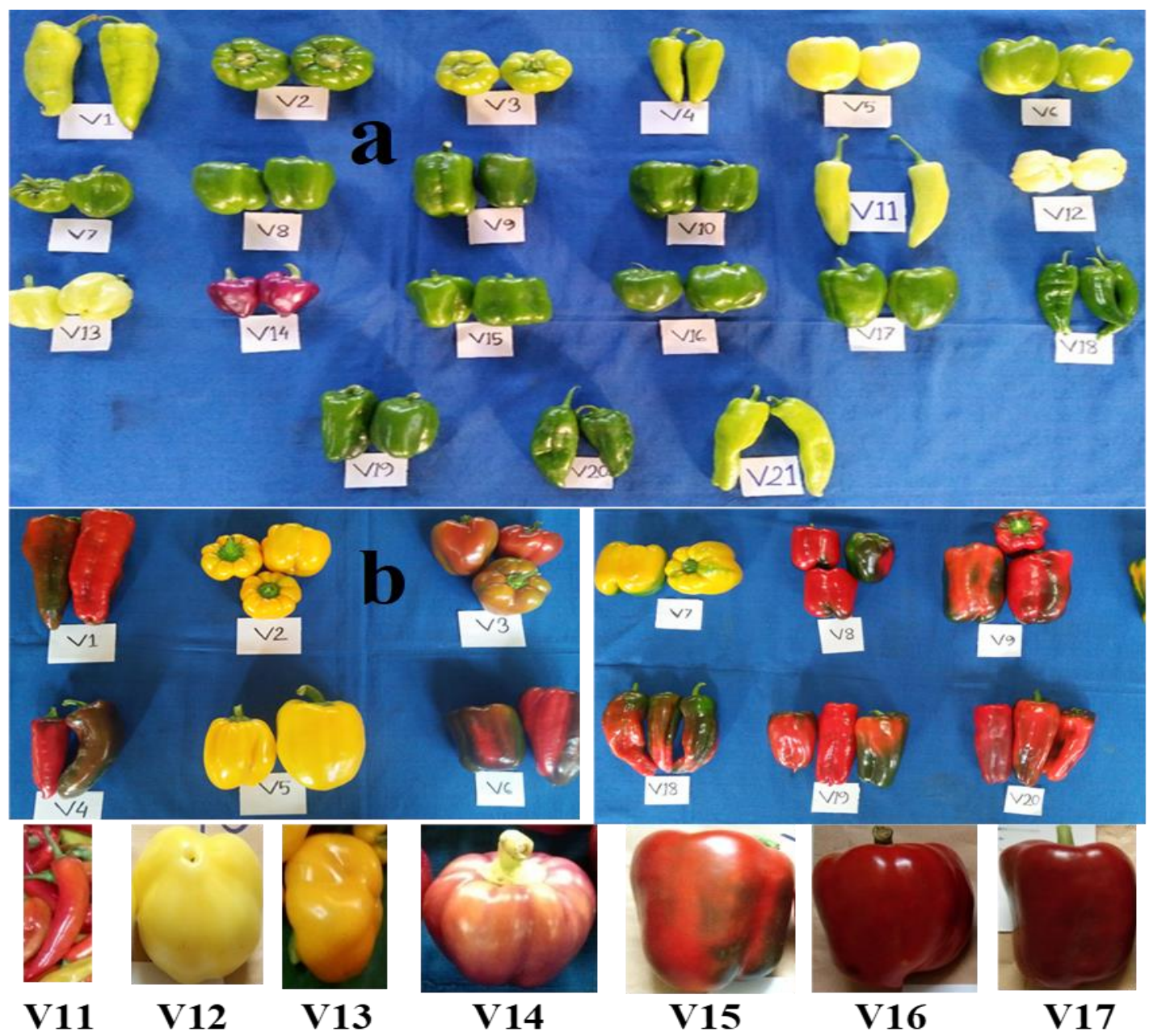

Fig. 1. Variation of fruit color, shape and size at (a) intermediate stage and (b) mature stage. (Here; V1- AVPP 0701; V2- AVPP 0402; V3- AVPP 0504; V4- AVPP 9807; V5- AVPP 0408; V6- AVPP 1112; V7- AVPP 1115; V8- AVPP 0019; V9- BARI M1; V10- BARI M2; V11- LS; V12- WBP 107; V13YEP 054; V14- PBP 057; V15- RBP 040; V16- PL 730; V17- CA 008; V18- CA 010; V19- CA 009; V20- CA 012 and V21-CA 011.). 
European Journal of Biology and Biotechnology www.ejbio.org

TABLE II: Morphological Characters Determining/Grading SCALE of SweEt Pepper GenOtYPeS

\begin{tabular}{|c|c|c|c|c|c|c|c|c|c|c|}
\hline Characters & & & & Deter & ing/grading s & & & & & \\
\hline Stem colour* & 1 Green & $\begin{array}{l}2 \text { Green with } \\
\text { purple streak }\end{array}$ & 3 Purple & $\begin{array}{l}\text { 4 Light green } \\
\text { with purple } \\
\text { streak }\end{array}$ & & & & & & \\
\hline $\begin{array}{c}\text { Nodal } \\
\text { anthocyanin* }\end{array}$ & 1 Green & 3 Light purple & 5 Purple & 7 Dark purple & & & & & & \\
\hline Stem shape* & 1 Cylindrical & 2 Angled & 3 Flattened & & & & & & & \\
\hline $\begin{array}{c}\text { Stem } \\
\text { pubescence* }\end{array}$ & 3 Sparse & 5 Intermediate & 7 Dense & & & & & & & \\
\hline $\begin{array}{l}\text { Plant growth } \\
\text { habit* }\end{array}$ & 3 Prostrate & $\begin{array}{l}5 \text { Intermediate } \\
\text { (compact) }\end{array}$ & 7 Erect & 9 Other & & & & & & \\
\hline $\begin{array}{c}\text { Branching } \\
\text { habit* }\end{array}$ & 3 Sparse & 5 Intermediate & 7 Dense & & & & & & & \\
\hline Leaf density* & 3 Sparse & 5 Intermediate & 7 Dense & & & & & & & \\
\hline Leaf colour* & 1 Yellow & 2 Light green & 3 Green & 4 Dark green & $\begin{array}{l}5 \text { Light } \\
\text { purple }\end{array}$ & 6 Purple & 7 Variegated & 8 Other & & \\
\hline Leaf shape* & 1 Deltoid & 2 Ovate & 3 Lanceolate & & & & & & & \\
\hline $\begin{array}{l}\text { Lamina } \\
\text { margin* }\end{array}$ & 1 Entire & 2 Undulate & 3 Ciliate & & & & & & & \\
\hline $\begin{array}{c}\text { Leaf } \\
\text { pubescence* }\end{array}$ & 3 Sparse & 5 Intermediate & 7 Dense & & & & & & & \\
\hline $\begin{array}{l}\text { Flower } \\
\text { position* }\end{array}$ & 3 Pendant & 5 Intermediate & 7 Erect & & & & & & & \\
\hline Corolla colour* & 1 White & 2 Light yellow & 3 Yellow & 4 Yellow-green & $\begin{array}{c}5 \text { Purple } \\
\text { with white } \\
\text { base }\end{array}$ & $\begin{array}{l}6 \text { White } \\
\text { with } \\
\text { purple } \\
\text { base }\end{array}$ & $\begin{array}{l}7 \text { White } \\
\text { with purple } \\
\text { margin }\end{array}$ & $\begin{array}{c}8 \\
\text { Purple }\end{array}$ & 9 Other & \\
\hline Anther colour* & 1 White & 2 Yellow & 3 Pale blue & 4 Blue & 5 Purple & 6 Other & & & & \\
\hline $\begin{array}{c}\text { Filament } \\
\text { colour* }\end{array}$ & 1 White & 2 Yellow & 3 Green & 4 Blue & $\begin{array}{l}5 \text { Light } \\
\text { purple }\end{array}$ & 6 Purple & & & & \\
\hline $\begin{array}{c}\text { Stigma } \\
\text { exertion* }\end{array}$ & 3 Inserted & 5 Same level & 7 Exserted & & & & & & & \\
\hline Male sterility* & 0 Absent & 1 Present & & & & & & & & \\
\hline $\begin{array}{l}\text { Fruit colour at } \\
\text { intermediate } \\
\text { stage* }\end{array}$ & 1 White & 2 Yellow & 3 Green & 4 Orange & 5 Purple & $\begin{array}{l}6 \text { Deep } \\
\text { purple }\end{array}$ & 7 Other & & & \\
\hline $\begin{array}{l}\text { Fruit colour at } \\
\text { mature stage* }\end{array}$ & 1 White & 2 Lemon-yellow & $\begin{array}{c}3 \text { Pale orange- } \\
\text { yellow }\end{array}$ & $\begin{array}{l}4 \text { Orange- } \\
\text { yellow }\end{array}$ & $\begin{array}{l}5 \text { Pale } \\
\text { orange and } \\
6 \text { Orange }\end{array}$ & $\begin{array}{l}7 \text { Light red } \\
\text { and } 8 \text { Red }\end{array}$ & 9 Dark red & $\begin{array}{c}10 \\
\text { Purple }\end{array}$ & $\begin{array}{c}11 \\
\text { Brown }\end{array}$ & $\begin{array}{l}12 \text { Black } \\
13 \text { Other }\end{array}$ \\
\hline Fruit shape* & 1 Elongate & 2 Almost round & 3 Triangular & 4 Campanulate & 5 Blocky & 6 Other & & & & \\
\hline $\begin{array}{l}\text { Fruit shape at } \\
\text { pedicel } \\
\text { attachment* }\end{array}$ & 1 Acute & 2 Obtuse & 3 Truncate & 4 Cordate & 5 Lobate & & & & & \\
\hline $\begin{array}{l}\text { Fruit shape at } \\
\text { blossom end* }\end{array}$ & 1 Pointed & 2 Blunt & 3 Sunken & $\begin{array}{l}4 \text { Sunken and } \\
\text { pointed }\end{array}$ & 5 Other & & & & & \\
\hline $\begin{array}{c}\text { Fruit blossom } \\
\text { end } \\
\text { appendage* }\end{array}$ & 0 Absent & 1 Present & & & & & & & & \\
\hline $\begin{array}{l}\text { Fruit cross- } \\
\text { sectional } \\
\text { corrugation* }\end{array}$ & $\begin{array}{l}3 \text { Slightly } \\
\text { corrugated }\end{array}$ & 5 Intermediate & 7 Corrugated & & & & & & & \\
\hline Seed colour* & $\begin{array}{c}1 \text { Straw (deep } \\
\text { yellow) }\end{array}$ & 2 Brown & 3 Black & 4 Other & & & & & & \\
\hline $\begin{array}{c}\text { Number of } \\
\text { seeds per fruit* }\end{array}$ & 1. $<20$ & 2. $20-50$ & 3. $>50$ & & & & & & & \\
\hline
\end{tabular}

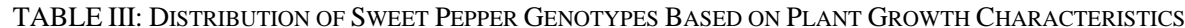

\begin{tabular}{|c|c|c|c|c|}
\hline Characters & Grading scale & $\begin{array}{c}\text { No. of } \\
\text { genotypes }\end{array}$ & Frequency $\%$ & Name of genotypes \\
\hline \multirow{4}{*}{ Stem colour } & 1 Green & 14 & 66.66 & $\begin{array}{c}\text { AVPP 0701, 0402, 0504, } 1115 \& \text { \& 0019; BARI M } 1 \text { \& 2; RBP 040; CA 0010, } \\
\text { 0011, 008, } 009 \text { \& 0012; LS. }\end{array}$ \\
\hline & $\begin{array}{l}2 \text { Green with } \\
\text { purple streak }\end{array}$ & 6 & 28.57 & AVPP 9807, 0408, 1112; WBP 107; YEP 054; PL 730 \\
\hline & $\begin{array}{l}4 \text { Light green with } \\
\text { purple streak }\end{array}$ & 1 & 4.76 & PBP 057 \\
\hline & 1 Green & 7 & 33.33 & BARI M 1 \& 2; YEP 054; RBP 040; CA 008, 0019 \& 0011 \\
\hline \multirow[t]{2}{*}{$\begin{array}{c}\text { Nodal } \\
\text { anthocyanin }\end{array}$} & 3 Light purple & 13 & 61.90 & $\begin{array}{c}\text { AVPP 0701, 0402, 0504, 9807, 1115, 0019, \& 1112; WBP 107; } \\
\text { PBP 057; PL 730; CA 0010, 0012; LS }\end{array}$ \\
\hline & 5 Purple & 1 & 4.76 & AVPP 0408 \\
\hline \multirow{2}{*}{ Stem shape } & 1 Cylindrical & 12 & 57.14 & $\begin{array}{c}\text { AVPP 9807, } 1112 \& \text { 0019; BARI M 1; WBP 107; YEP 054; PBP 057; CA } \\
0010,0011,008 \text { \& 009; LS }\end{array}$ \\
\hline & 2 Angled & 9 & 42.85 & $\begin{array}{c}\text { AVPP 0701, 0402, 0504, } 0408 \& \text { 1115; BARI M 2; RBP 040; YEP 054; CA } \\
0012\end{array}$ \\
\hline \multirow{3}{*}{$\begin{array}{l}\text { Stem } \\
\text { pubescence }\end{array}$} & 3 Sparse & 15 & 71.42 & AVPP 0408; WBP 107; YEP 054; PL 730; LS \\
\hline & 5 Intermediate & 2 & 9.52 & $\begin{array}{c}\text { AVPP 0402, 0504, 9807, 1115, } 0019 \& \text { \& 1112; BARI M 1; RBP 040; CA 008, } \\
0010 \& 0011,\end{array}$ \\
\hline & 7 Dense & 4 & 19.04 & AVPP 0701, BARI M 1; PBP 057; CA 009 \\
\hline \multirow{3}{*}{$\begin{array}{l}\text { Plant growth } \\
\text { habit }\end{array}$} & 3 Prostrate & 3 & 14.28 & BARI M 2; CA 0010, 0012 \\
\hline & 5 Intermediate & 13 & 61.90 & $\begin{array}{c}\text { AVPP 0701, 1112, 0402,0019 \& 1115; BARI M 1; WBP 107; YEP 054; PBP } \\
\text { 057; RBP 040; CA 008; PL } 730\end{array}$ \\
\hline & 7 Erect & 5 & 23.80 & AVPP $0504 \& 0408 ;$ CA $009 \& 0011 ;$ LS \\
\hline \multirow{2}{*}{$\begin{array}{l}\text { Branching } \\
\text { habit }\end{array}$} & 3 Sparse & 4 & 19.09 & AVPP 0504; PL 730; CA 009, 0011 \\
\hline & 5 Intermediate & 14 & 66.66 & $\begin{array}{c}\text { AVPP 0701, 0402, 1112, 9807, 0408, } 1115 \text { \& 0019; BARI M } 1 \& \text { \&; RBP 040; } \\
\text { CA 008, 0010 \& 0011; LS }\end{array}$ \\
\hline
\end{tabular}




$\begin{array}{cccc} & \text { 7 Dense } & 3 & 33.33 \\ \text { Leaf density } & \text { 3 Sparse } & 4 & 14.28 \\ & \text { 5 Intermediate } & 5 & 23.80 \\ & 7 \text { Dense } & 10 & 47.61 \\ \text { Leaf colour } & 3 \text { Green } & 5 & 23.80 \\ & \text { 4 Dark green } & 16 & 76.19 \\ \text { Leaf shape } & \text { 1 Deltoid } & 12 & 57.14 \\ & \text { 2 Ovate } & 2 & 9.52 \\ \text { Lamina } & \text { 3 Lanceolate } & 7 & 33.33 \\ \text { margin } & \text { 1 Entire } & 21 & 100 \\ \text { Leaf } & & & \\ \text { pubescence } & 3 \text { Sparse } & 21 & 100\end{array}$

RBP 057; WBP 107; YEP 054;

AVPP 0504 \& 9807, LS; CA 009

AVPP 0408, 1112; BARI M 2; PL 730; CA 0010

AVPP 0701, 0402, 1115 and 0019; BARI M 1; WBP 107; YEP 054; PBP 057 ; RBP 040; CA 008

AVPP 9807; WBP 107; YEP 054; PBP 057; CA 008

AVPP 0701, 0402, 0504, 1115, 0019, 0408 \& 1112; BARI M 1 \& 2; RBP 040; PL 730; CA 0010, 0011, CA 009 \& 0012; LS

AVPP 0701 \& 0019; BARI M 1 \& 2; YEP 054; PBP 057; PL 730; CA 008, 0011, 009 \& 0012; LS

AVPP 9807; CA 0010

AVPP 0701, 0402, 0504, 0408, 1112 \& 1115; WBP 107; RBP 040;

AVPP 0701, 0402, 0504, 1115, 0019, 9807, 0408 \& 1112; BARI M 1 \& 2; RBP 040; WBP 107; YEP 054; PL 730; PBP 057; CA 008, 009, 0010, 0011 \& 0012; LS

AVPP 0701, 0402, 0504, 1115, 0019, 9807, 0408 \& 1112; BARI M 1 \& 2; RBP 040; WBP 107; YEP 054; PL 730; PBP 057; CA 008, 009, 0010, 0011 \& 0012; LS

\begin{tabular}{|c|c|c|c|c|}
\hline Characters & Grading scale & $\begin{array}{l}\text { No. of } \\
\text { genotypes }\end{array}$ & Frequency $\%$ & Name of genotypes \\
\hline \multirow{3}{*}{ Flower position } & 3 Pendent & 8 & 38.09 & AVPP 0701, 0504, 9807, 0408 \& 1112; LS; CA 0010 \& 0012 \\
\hline & 5 Intermediate & 11 & 52.38 & $\begin{array}{l}\text { AVPP } 1115 \& \text { 0019; BARI M } 1 \& \text { 2; PL 730; WBP 107; YEP 054; CA 008, } 009 \& \text { \& } \\
0011\end{array}$ \\
\hline & 7 Erect & 2 & 9.52 & AVPP 0402, RBP 040; \\
\hline \multirow[t]{2}{*}{ Corolla colour } & 1 White & 21 & 100 & $\begin{array}{l}\text { AVPP 0701, 0402, 0504, 1115, 0019, 9807, } 0408 \& \text { 1112; BARI M } 1 \& \text { \& } \text { RBP } \\
\text { 040; WBP 107; YEP 054; PL 730; PBP 057; CA 008, 009, 0010, } 0011 \& \text { 0012; LS }\end{array}$ \\
\hline & 1 White & 5 & 23.80 & AVPP 0701; BARI M 1; YEP 054; CA 0011; LS \\
\hline \multirow{4}{*}{ Anther color } & 2 Yellow & 2 & 9.52 & AVPP 9807; BARI M 2 \\
\hline & 3 Pale blue & 4 & 19.04 & AVPP 0402 \& 0019; RBP 040; CA 009 \\
\hline & 4 Blue & 8 & 38.09 & AVPP 0504, 1115, $0408 \&$ 1112; WBP 107; PL 730; CA 008 \& 0010 \\
\hline & 5 Purple & 2 & 9.52 & PBP 057 \\
\hline \multirow[t]{3}{*}{ Filament color } & 1 White & 15 & 71.42 & $\begin{array}{c}\text { AVPP 0701, 0504, 1115, 9807, } 0408 \& \text { \& 1112; BARI M 1, WBP 107; PL 730; PBP } \\
\text { 057; CA 008, 0010, } 0011 \& \text { 0012; LS }\end{array}$ \\
\hline & 2 Yellow & 6 & 28.57 & AVPP 0402 \& 0019; BARI M 2; YEP 054; RBP 040; CA 009 \\
\hline & 3 Inserted & 4 & 19.04 & AVPP 0408; WBP 107; PL 730 \\
\hline \multirow[t]{2}{*}{$\begin{array}{l}\text { Stigma } \\
\text { exsertion }\end{array}$} & 5 Same level & 13 & 61.90 & $\begin{array}{l}\text { AVPP 0402, 0504, 1115, } 1112 \text { \& 0019; BARI M 2; LS; WBP 107; CA 008, 0010, } \\
0011 \& 0012\end{array}$ \\
\hline & 7 Exserted & 4 & 19.04 & AVPP 0701; BARI M 1; PBP 057; CA 008 \\
\hline \multirow[t]{2}{*}{ Male sterility } & 0 Absent & 21 & 100 & $\begin{array}{l}\text { AVPP 0701, 0402, 0504, 1115, 0019, 9807, } 0408 \text { \& 1112; BARI M } 1 \& \text { \& } \text { RBP } \\
\text { 040; WBP 107; YEP 054; PL 730; PBP 057; CA 008, 009, 0010, } 0011 \text { \& 0012; LS }\end{array}$ \\
\hline & 2 Yellow & 4 & 19.04 & AVPP 0402 \& 0408; WBP 107; YEP 054 \\
\hline \multirow{4}{*}{$\begin{array}{c}\text { Fruit color at } \\
\text { intermediate } \\
\text { stage }\end{array}$} & 3 Green & 9 & 42.85 & AVPP 0019; BARI M 1 \& 2; RBP 040; PL 730; CA 008, 009, 0010 \& 0012 \\
\hline & 5 Deep purple & 1 & 4.76 & PBP 057 \\
\hline & 7 Light green & 5 & 23.80 & AVPP $0701,1115,9807 \&$ 1112; CA 0011 \\
\hline & 8 Light yellow & 2 & 9.52 & AVPP 0504; LS \\
\hline \multirow{5}{*}{$\begin{array}{l}\text { Fruit colour at } \\
\text { mature stage }\end{array}$} & 2 Lemon Yellow & 2 & 9.52 & AVPP $0402 \& 1115$ \\
\hline & 4 Orange yellow & 3 & 14.28 & BARI M 2; WBP 107; YEP 054 \\
\hline & 6 Orange & 1 & 4.72 & PBP 057 \\
\hline & 8 Red & 14 & 66.66 & $\begin{array}{c}\text { AVPP 0701, 0504, 9807, } 1112 \& \text { 0019; BARI M 1; LS; RBP 40; PL 730; CA 008, } \\
\text { 009, 0010, } 0011 \& 0012\end{array}$ \\
\hline & 13 Deep yellow & 1 & 4.72 & AVPP 0408 \\
\hline \multirow{5}{*}{ Fruit shape } & 1 Elongate & 2 & 9.52 & CA $0010 \& 0011$ \\
\hline & 3 Triangular & 3 & 14.28 & AVPP 0701; LS; CA 0012 \\
\hline & 4 Campanulate & 7 & 33.33 & AVPP 9807, 0408, 1115, 1112; WBP107; YEP 054; PBP 057 \\
\hline & 5 Blocky & 8 & 38.09 & AVPP 0402 \& 0019; BARI M 1 \& 2; RBP 040; PL 730; CA 008 \& 009 \\
\hline & 6 Tomato & 1 & 4.72 & AVPP 0504 \\
\hline \multirow{3}{*}{$\begin{array}{l}\text { Fruit shape at } \\
\text { blossom end }\end{array}$} & 1 Pointed & 5 & 23.80 & AVPP $0701 \&$ 9807; LS; PBP 057; CA 0011 \\
\hline & 2 Blunt & 3 & 14.28 & AVPP 0504; CA $0010 \& 0011$ \\
\hline & 3 Sunken & 13 & 61.90 & $\begin{array}{c}\text { AVPP 0402, 1115, 0402, 0504, \& 0019; BARI M } 1 \& \text { 2; WBP 107; YEP 054; RBP } \\
\text { 040; PL 730; CA } 008 \& 009\end{array}$ \\
\hline \multirow{3}{*}{$\begin{array}{c}\text { Fruit shape at } \\
\text { pedicel } \\
\text { attachment }\end{array}$} & 2 Obtuse & 3 & 14.28 & CA $0010 \& 0011 ;$ LS \\
\hline & 3 Truncate & 4 & 19.04 & AVPP 0701, 9807; PBP 057; CA 0012 \\
\hline & 5 Lobate & 14 & 66.66 & $\begin{array}{c}\text { AVPP 0402, 0504, 1115, 0019, } 0408 \text { \& 1112; BARI M } 1 \text { \& 2; WBP 107; YEP } \\
\text { 054; PL 730; RBP 040; CA } 008 \text { \& } 009\end{array}$ \\
\hline \multirow{2}{*}{$\begin{array}{l}\text { Fruit blossom } \\
\text { end appendage }\end{array}$} & 0 Absent & 20 & 95.23 & $\begin{array}{l}\text { AVPP 0701, 0402, 0504, 1115, 0019, 9807, } 0408 \& \text { 1112; BARI M } 1 \& \text { \& RBP } \\
\text { 040; WBP 107; YEP 054; PL 730; CA 008, 009, 0010, 0011 \& 0012; LS }\end{array}$ \\
\hline & 1 Present & 1 & 4.72 & PBP 057 \\
\hline \multirow{3}{*}{$\begin{array}{l}\text { Fruit cross- } \\
\text { sectional } \\
\text { corrugation }\end{array}$} & 3 Slightly corrugated & 3 & 14.28 & AVPP 9807; LS; CA 0011 \\
\hline & 5 Intermediate & 3 & 14.28 & AVPP 0701; PBP 057; CA 0010 \\
\hline & 7 Corrugated & 15 & 71.42 & $\begin{array}{c}\text { AVPP 0402, 0504, 1115, 0408, } 1112 \text { \& 0019; BARI M } 1 \text { \& 2; WBP 107; YEP } \\
\text { 054; PL 730; RBP 040; CA 008, } 009 \text { \& } 0012\end{array}$ \\
\hline Seed colour & 1 Straw & 21 & 100 & $\begin{array}{l}\text { AVPP 0701, 0402, 0504, 1115, 0019, 9807, } 0408 \& \text { 1112; BARI M } 1 \& \text { \& } \text { RBP } \\
\text { 040; WBP 107; YEP 054; PL 730; PBP 057; CA 008, 009, 0010, } 0011 \& \text { 0012; LS }\end{array}$ \\
\hline \multirow{2}{*}{$\begin{array}{l}\text { Number of } \\
\text { seeds per fruit }\end{array}$} & $220-50$ & 4 & 19.04 & AVPP 0402, 0504, 1115, 1112; YEP 054; \\
\hline & $3>50$ & 17 & 80.95 & $\begin{array}{c}\text { AVPP 0701, 0019, } 9807 \text { and 0408; BARI M } 1 \& \text { \& 2; RBP 040; WBP 107; PL 730; } \\
\text { PBP 057; LS; CA 008, 009, 0010, 0011, 0012 }\end{array}$ \\
\hline
\end{tabular}




\section{CONCLUSIONS}

Based on the above discussion it can be concluded that a distinct morphological variation was observed among twenty-one sweet pepper genotypes. Among different morphological traits studied, a higher frequency was observed for nodal anthocyanin, dark green leaves, intermediate branching habit and flower position, blocky fruit shape, green and red color fruit, sunken blossom end shape etc. indicating fitness of genotypes. The study suggested that the genotypes like AVPP 0701, 0504, 0408 and 0019; BARI M 1; CA 008 exhibited distinct variation in various aspects while PBP 057 having purple color can be used in future breeding programme for the development of superior open pollinated varieties or hybrids in Bangladesh for higher yield and quality improvement of sweet pepper.

\section{ACKNOWLEDGMENT}

The first author acknowledges the Bangabandhu Science and Technology Fellowship Trust by the Ministry of Science \& Technology (MoST), Government of the People's Republic of Bangladesh. The Authors also acknowledges Bangabandhu Sheikh Mujibur Rahman Agricultural University, Gazipur 1706, Bangladesh for technical support for this study.

\section{COMPETING INTERESTS}

Authors have declared that no competing interests exist.

\section{REFERENCES}

[1] R. Negi, S. Thakur, \& P. Sharma, "Advances in the breeding of bell pepper-a review”, Intl J Curr Microbiol App Sci, 7(4), pp. 2272-2281, 2018.

[2] W. H. Greenleaf, "Pepper Breeding. In: Bassett, M. J. (ed) Breeding Vegetable Crops", Avi Publishing, Westport, Connecticut, pp. 67-134, 1986.

[3] M. C. Joshi, and D. P. Singh, "Chemical Composition in Bell Pepper", Indina Hort. 20, pp. 19-21, 1975.

[4] C. Chávez-Mendoza, E. Sanchez, E. Muñoz-Marquez, J. P. SidaArreola, and M. A. Flores-Cordova, "Bioactive compounds and antioxidant activity in different grafted varieties of bell pepper", Antioxidants, 4(2), pp. 427-446, 2015.

[5] J. Ferdousi, Z. A. Riyadh, M. I. Hossain, S. R. Saha, \& M. Zakaria, "Mushroom Production Benefits, Status, Challenges and Opportunities in Bangladesh: A Review", Annual Research \& Review in Biology, 34(6), pp. 1-1, 2020.

[6] ICDDRB (International Centre for Diarrheal Disease Research in Bangladesh), "A brief guide to malnutrition and its impact globally and in Bangladesh", 2019. (Accessed on 12 December 2019) Available:https://www.icddrb.org/newsand-events/presscorner/mediaresources/malnutrition.

[7] BBS (Bangladesh Bureau of Statistics), "Yearbook of Agricultural Statistics: Ministry of Planning, Government of the People's Republic of Bangladesh", Dhaka, Bangladesh, pp. 139, 2020.

[8] Akter, M. R. Ali, T. Hasan, G. M. A. Halim, and H. Mehraj, "Effect of Planting Time and Seedling Raising Methods on Growth and Yield of Capsicum”, EC Nutrition 7.4, pp. 179-184, 2017.

[9] BARI (Bangladesh Agricultural Research Institute), "Handbook on Agro-Technology, $8^{\text {th }}$ edition", Gazipur-1706, p. 549, 2019.

[10] N. U. Mahmud, R. Chakma, N. U. Ahmed, M. A. U. Zaman, and A. Hossain, "Effect of sowing date on quality seed production of sweet pepper in Bangladesh", Progressive Agriculture, 28(3), pp. 216-221, 2017.

[11] M. B. Taylor, and V. S. Tuia, "The Importance of Germplasm Development and Exchange in Meeting the Challenges of the 21st
Century in the Pacific Region", In VI International Symposium on In Vitro Culture and Horticultural Breeding 829, pp. 231-239, 2008

[12] D. Gogoi, and B. P. Gautam, "Evaluation of chilli (Capsicum spp.) germplasm for fruit yield and component characters" J. Appl. Hort, 4(1), pp. 41-442002.

[13] M. Sreenivas, A. B. Sharangi, S. Banerjee, T. Bhattacharya, P. K. Maurya, and A. Chattopadhyay, "Characterization and evaluation of indigenous and exotic genotypes for identification of spice chilli lines", IJCS, 7(2), pp. 1830-1837, 2019

[14] U. Joshi, D. K. Rana, V. Singh, and R. Bhatt, "Morphological characterization of chilli (Capsicum annum L.) genotypes", J Emerg Tech Inn Res, 7, pp. 710, 2020.

[15] M. E. Osawaru, M. C. Ogwu, and J. Omologbe, "Characterization of three Okra [Ablemoschus (L.)] accessions using morphology and SDSPAGE for the basis of conservation", Egyptian Academic Journal of Biological Science, 5(1), pp. 55-65, 2014

[16] B. P. Luitel, N. Y. H. C. Ro, Ko, J. S. Sung, J. H. Rhee, and O. S. Hur, "Phenotypic Variation in a Germplasm Collection of Pepper (Capsicum chinense Jacq.) from Korea", Journal of Crop Science and Biotechnology, 21(5), pp. 499-506, 2018.

[17] J. C. Smith, and O. S. Smith, "The description and assessment of distances between inbred lines of maize, II: The utility of morphological biochemical, and genetic descriptors and a scheme for the testing of distinctiveness between inbred lines", Maydica, 34(2), pp. 151-161, 1989.

[18] M. M. R. Salim, M. H. Rashid, M. M. Hossain, and M. Zakaria, "Morphological characterization of tomato (Solanum lycopersicum L.) genotypes", Journal of the Saudi Society of Agricultural Sciences, 19(3), pp. 233-240, 2020.

[19] S. G. Agong, S. Schittenhelm, and W. Friedt, "Genotypic variation of Kenyan tomato (Lycopersicon esculentum L.) germplasm”, Journal of food technology in Africa, 6(1), pp. 13-17, 2001.

[20] P. R. Dharmatti, B. B. Madalgeri, I. M. Mannikeri, R. V. Patil, \& G. Patil, "Genetic divergence studies in summer tomatoes", Karnataka Journal of Agricultural Sciences, 14(2), pp. 407-411, 2001.

[21] V. A. Parthasarathy, and C. Aswath, "Genetic diversity among tomato genotypes", Indian Journal of Horticulture, 59(2), pp. 162-166, 2002.

[22] Anonymous, "Agro-cimatarologicaldata. Agromet Division. Bangladesh Meteorological Department, Joydebpur, Gazipur”, pp. 35 $65,1995$.

[23] FAO (Food and Agricultural Organization), Production Year Book, Food and Agricultural Organization of the United Nations, Rome Italy", 1988

[24] J. Haider, T. Maruomoto and A. K. Azad, "Estimation of microbial biomass, carbon and nitrogen in Bangladesh Soils", Sci. Plant Nutr. 37(4), pp. 591-599, 1991.

[25] T.G. Berke, L.L. Black, S.K. Green, R.A. Morris, N.S. Tlekar, and J.F Wang, "Suggested Cultural Practices for Field Cultivation of Sweet Pepper”, Int. Cooperation's Guide. AVRDC, Pub. No. 99-497, AVRDC, Shanhua, Taiwan, 741, ROC, pp. 197, 1999.

[26] IPGRI, AVRDC and CATIE, "Descriptors for Capsicum (Capsicum spp.) IPGRI, Rome, Italy", 1995.

[27] S. Farwah, K. Hussain, K. P. Wani, Z. A. Dar, Z. A. Bhat, S. Rizvi, and S. Maqbool, "Distinctiveness, uniformity and stability characterization of Chilli (Capsicum annuum L.) genotypes under temperate conditions of Kashmir", SKUAST Journal of Research, 21(2), pp. 152-165, 2019.

[28] J. K. Moon, and T. Shibamoto, "Antioxidant assays for plant and food components", Journal of agricultural and food chemistry, 57(5), pp. 1655-1666, 2009.

[29] S. Sonia, and S. Ruchi, "Morphological characterization of bell pepper (Capsicum annuum var. grossum) genotypes and their application for distinctness, uniformity and stability testing", Indian Journal of Agricultural Sciences, 81(3), pp. 240-246, 2011.

[30] M. K. Bozokalfa,and D. Eşiyok, "Evaluation of morphological and agronomical characterization of Turkish pepper accessions", International journal of vegetable science, 17(2), pp. 115-135, 2011.

[31] R. Pachiyappan, and K. Saravannan, "Studies on genetic variability and correlation for fruit yield and fruit quantity characters of okra", Asian Journal of Horticulture, 11(1), pp. 101-104, 2016.

[32] L. P. Peña-Yam, L. S. Muñoz-Ramírez, S. A. Avilés-Viñas, A. CantoFlick, A. Guzmán-Antonio, and N. Santana-Buzzy, "Floral biology studies in habanero pepper (Capsicum chinense jacq.) to implement in a cross-breeding program", Agriculture, 9(12), pp. 249, 2019.

[33] S. Rahman, M. A. Hossain, and R. Afroz, "Morphological characterization of chilli germplasm in Bangladesh", Bangladesh Journal of Agricultural Research, 42(2), pp. 207-219, 2017.

[34] J. Rajesh, V. M. Prasad, and A. Kerketta, "Evaluation of Different Okra [Abelmoschus esculentus (L.) Moench] Hybrids for Yield and Yield Attributes under Allahabad", Agro-climatic Condition. Int. J. Pure App. Biosci, 6(5), pp. 1343-1346, 2018 
[35] J. O. Uzo, "Hybrid vigour and gene action of two qualitative traits of flavour peppers in Nigeria", Scientia horticulturae, 22(4), pp. 321-326, 1984.

[36] S. Sood, and N. Kumar, "Morphological studies of bell pepper germplasm", International journal of vegetable science, 17(2), pp. 144$156,2011$.

[37] V. Sharma, S. Sood, V. K. Sood, and Y. Singh, "Morphological characterization of Bell Pepper (Capsicum аппиит L. var. grossum Sendt.) genotypes", Himachal Journal of Agricultural Research, 43(1), pp. 33-39, 2017

[38] V. K. Sharma, A. Srivastava, and M. Mangal, "Recent Trends in Sweet Pepper Breeding", In Accelerated Plant Breeding, Vol. 2, pp. 417-444, Springer, Cham, 2020. 